\title{
AN EPIDEMIC MODEL FOR THE TRANSMISSION DYNAMICS OF HIV AND ANOTHER PATHOGEN
}

\author{
S. M. MOGHADAS' ${ }^{1}$ and A. B. GUMEL ${ }^{1}$
}

(Received 12 October, 2001; revised 23 April, 2002)

\begin{abstract}
A five-dimensional deterministic model is proposed for the dynamics between HIV and another pathogen within a given population. The model exhibits four equilibria: a diseasefree equilibrium, an HIV-free equilibrium, a pathogen-free equilibrium and a co-existence equilibrium. The existence and stability of these equilibria are investigated. A competitive finite-difference method is constructed for the solution of the non-linear model. The model predicts the optimal therapy level needed to eradicate both diseases.
\end{abstract}

\section{Introduction}

Following the discovery of the human immunodeficiency virus (HIV) three decades ago, much attention has been focussed on the development and analysis of realistic mathematical models for the transmission dynamics, pathogenesis and control mechanisms of HIV (see, for instance, [1, 2, 4, 5, 7]).

Since HIV is known to replicate only in activated CD4+ T cells (see $[4,5,7])$, the study of the effect of the interactions between HIV and other pathogens (such as the flu virus, mycobacterium tuberculosis, the hepatitis virus etc.) is important. McLean and Nowak [5] have proposed within host (in vivo) models for the dynamics between $\mathrm{HIV}$ and activated $\mathrm{CD} 4+\mathrm{T}$ cells specific to other pathogens.

Our study is focussed on the design and analysis of a new population model for the transmission dynamics of HIV and another competing pathogen (assumed to be curable) within a given population. Such an epidemiological model can be used to assess anti-HIV programmes in nations where access to highly-active antiretroviral therapy (HAART) [7] for controlling HIV is not readily available, but cures for the competing pathogens (causing tuberculosis, flu, hepatitis etc.) are generally

\footnotetext{
'Department of Mathematics, University of Manitoba, Winnipeg, Manitoba R3T 2N2, Canada; e-mail: moghadas@cc.umanitoba.ca and gumelab@cc.umanitoba.ca.

(C) Australian Mathematical Society 2003, Serial-fee code 1446-1811/03
} 
available and affordable. The central question to ask is whether an effective treatment mechanism that solely focuses on eradicating competing pathogens can reduce the spread of HIV within the population.

In this paper, we propose a new deterministic model for the transmission dynamics of $\mathrm{HIV}$ and a single competing pathogen that is curable. Qualitative and numerical analyses of the resulting five-dimensional nonlinear model are carried out to determine the optimal therapeutic coverage levels needed for eradicating both diseases. The model is formulated in Section 2 and analysed in Section 3. The effect of treatment on the stability of the associated equilibria is discussed in Section 4. In Section 5, we construct a robust numerical method, which is free of scheme-dependent numerical instabilities (such as oscillations, chaos, bifurcations and convergence to spurious zeros). Numerical experiments are reported in Section 6.

\section{Mathematical model}

The model monitors five populations: susceptibles $(X)$, individuals infected with a curable pathogen $\left(Y_{1}\right)$, HIV-infected individuals $\left(Y_{2}\right)$, HIV-infected individuals that have progressed to clinical AIDS $(A)$ and the population of individuals infected with the pathogen who become non-infectious following effective treatment $(Z)$. The total population size is $N=N(t)=X(t)+Y_{1}(t)+Y_{2}(t)+A(t)+Z(t)$.

2.1. Susceptible individuals, $X(t)$. All individuals recruited into the population at a rate of $\Pi$ per year are considered to be susceptible to both infectious diseases (HIV and the other pathogen). Susceptibles die of natural causes at a rate of $\mu$ per year. Susceptibles are lost following contact with members of the $Y_{i}$ populations $(i=1,2)$ at rate $\beta_{i}$, respectively. The average number of contacts per unit time is denoted by $c$. Thus

$$
\frac{d X}{d t}=\Pi-\mu X-\left(\frac{c \beta_{1} Y_{1}+c \beta_{2} Y_{2}}{N}\right) X
$$

2.2. Pathogen-infected individuals, $Y_{1}(t)$. This population is generated following the infection of susceptibles with the pathogen at rate $\beta_{1}$. Since members of this $\left(Y_{1}\right)$ population can be cured, we model the effect of treatment in terms of inhibition of transmission probability $\beta_{1}$ given by $(1-\tau) \beta_{1}$. This population is diminished by HIV infection (at rate $\beta_{2}$ ) and by natural death (at rate $\mu$ ). This gives

$$
\frac{d Y_{1}}{d t}=\frac{(1-\tau) c \beta_{1} Y_{1} X}{N}-\frac{c \beta_{2} Y_{1} Y_{2}}{N}-\mu Y_{1}
$$

2.3. HIV-infected individuals, $Y_{2}(t)$. This population is generated by the HIV infection of both the susceptibles and pathogen-infected individuals (at rate $\beta_{2}$ ). It 
is diminished by natural death (at rate $\mu$ ) and by the development of clinical AIDS (at rate $v$ ). For computational convenience, it is assumed that $Y_{2}$ individuals are not susceptible to infection by the pathogen. It is further assumed that anti-HIV treatment is not available within the community; hence members of the $Y_{2}$ and $A$ populations are not treated. This suggests

$$
\frac{d Y_{2}}{d t}=\frac{c \beta_{2} Y_{2} X}{N}+\frac{c \beta_{2} Y_{1} Y_{2}}{N}-(\mu+v) Y_{2}
$$

2.4. Individuals with clinical AIDS, $A(t)$. The population of individuals with clinical AIDS, $A(t)$, increases when members of the $Y_{2}$ population progress to clinical AIDS (at rate $v$ ). This population is reduced by natural death (at rate $\mu$ ) and by AIDS-induced death (at rate $d$ ). Thus

$$
\frac{d A}{d t}=v Y_{2}-(\mu+d) A \text {. }
$$

2.5. Non-infectious individuals, $Z(t)$. This population is generated following the effective treatment of members of the $Y_{1}$ population (at a rate $\tau$ ) and is diminished by natural death (at a rate $\mu$ ), so that

$$
\frac{d Z}{d t}=\frac{\tau c \beta_{1} Y_{1} X}{N}-\mu Z
$$

In summary, the model consists of the equations

$$
\begin{aligned}
\frac{d X}{d t} & =\Pi-\mu X-\left(\frac{c \beta_{1} Y_{1}+c \beta_{2} Y_{2}}{N}\right) X, \\
\frac{d Y_{1}}{d t} & =\frac{(1-\tau) c \beta_{1} Y_{1} X}{N}-\frac{c \beta_{2} Y_{1} Y_{2}}{N}-\mu Y_{1}, \\
\frac{d Y_{2}}{d t} & =\frac{c \beta_{2} Y_{2} X}{N}+\frac{c \beta_{2} Y_{1} Y_{2}}{N}-(\mu+v) Y_{2}, \\
\frac{d A}{d t} & =v Y_{2}-(\mu+d) A, \\
\frac{d Z}{d t} & =\frac{\tau c \beta_{1} Y_{1} X}{N}-\mu Z .
\end{aligned}
$$

It should be mentioned that since the physical situation being modelled is that of population dynamics, it is necessary to impose the condition that all the dependent variables and parameters of the model are non-negative.

\section{Existence and stability of equilibria}

Defining, first of all, the force of infection given by (see $[1,8])$

$$
G(t)=\frac{c \beta_{1} Y_{1}(t)+c \beta_{2} Y_{2}(t)}{N(t)}, \quad H(t)=\frac{c \beta_{2} Y_{2}(t)}{N(t)},
$$


it follows from (2.1) that the associated expressions for the population densities at equilibrium are (see $[1,8])$

$$
\begin{array}{lll}
X^{*}=\frac{\Pi}{\mu+G^{*}}, & Y_{1}^{*}=\frac{(1-\tau)\left(G^{*}-H^{*}\right) X^{*}}{\mu+H^{*}}, & Y_{2}^{*}=\frac{H^{*}\left(X^{*}+Y_{1}^{*}\right)}{\mu+v}, \\
A^{*}=\frac{v Y_{2}^{*}}{\mu+d}, & Z^{*}=\frac{\tau\left(G^{*}-H^{*}\right) X^{*}}{\mu} \text { and } & N^{*}=\frac{1}{\mu}\left(\Pi-d A^{*}\right) .
\end{array}
$$

Substituting the above into the expressions for $G$ and $H$ in (3.1) gives

$$
G^{*}=\frac{1}{N^{*}}\left(\frac{c \beta_{1}(1-\tau) \Pi\left(G^{*}-H^{*}\right)}{\left(\mu+H^{*}\right)\left(\mu+G^{*}\right)}+\frac{c \beta_{2} H^{*}}{\mu+v}\left[\frac{\Pi}{\mu+G^{*}}+\frac{(1-\tau) \Pi\left(G^{*}-H^{*}\right)}{\left(\mu+H^{*}\right)\left(\mu+G^{*}\right)}\right]\right)
$$

and

$$
H^{*}=\frac{1}{N^{*}}\left(\frac{c \beta_{2} H^{*}}{\mu+v}\left[\frac{\Pi}{\mu+G^{*}}+\frac{(1-\tau) \Pi\left(G^{*}-H^{*}\right)}{\left(\mu+H^{*}\right)\left(\mu+G^{*}\right)}\right]\right) .
$$

To find the equilibria of the model, we need to determine the fixed points of the equation

$$
x=\Phi(x)=\left(\begin{array}{l}
\phi_{1}(G, H) \\
\phi_{2}(G, H)
\end{array}\right),
$$

where $\phi_{1}$ and $\phi_{2}$ are the right-hand sides of (3.2) and (3.3), respectively.

3.1. Disease-free equilibrium. Clearly $(0,0)$ is a fixed point of $\Phi$ (which corresponds to the disease-free equilibrium of the model) since $\phi_{1}(0,0)=0$ and $\phi_{2}(0,0)=0$. In the context of $(2.1)$, this fixed point corresponds to

$$
E_{0}=(\Pi / \mu, 0,0 ; 0,0) \text {. }
$$

The Jacobian of $\Phi$ is given by

$$
J=\left(\begin{array}{ll}
\partial \phi_{1}(G, H) / \partial G & \partial \phi_{1}(G, H) / \partial H \\
\partial \phi_{2}(G, H) / \partial G & \partial \phi_{2}(G, H) / \partial H
\end{array}\right) .
$$

Evaluating $J$ at $(0,0)$ gives

$$
J_{0}=\left(\left.\begin{array}{cc}
R_{1} & \frac{\partial \phi_{1}(G, H)}{\partial H} \\
0 & R_{2}
\end{array}\right|_{(0,0)}\right),
$$

where

$$
R_{1}=c \beta_{1}(1-\tau) / \mu \text { and } R_{2}=c \beta_{2} /(\mu+v) .
$$

The eigenvalues of $J_{0}$ are $R_{1}$ and $R_{2}$. The dominant eigenvalue of $J_{0}$, given by $\mathscr{R}=\max \left\{R_{1}, R_{2}\right\}$, is the basic reproductive number $[1,3,8]$. It follows then that the disease-free equilibrium is locally asymptotically stable if $\mathscr{R}<1$ and unstable if $\mathscr{R}>1$. 


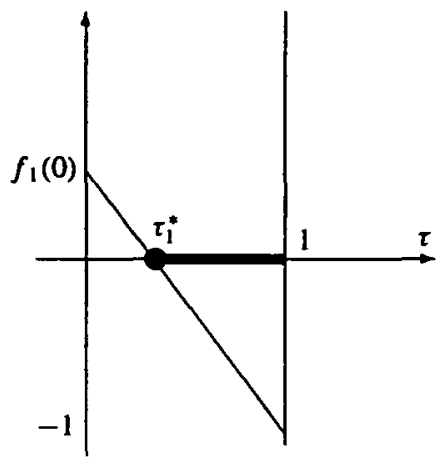

FIGURE 1. $f_{1}(0)=c \beta_{1} / \mu-1>0$.

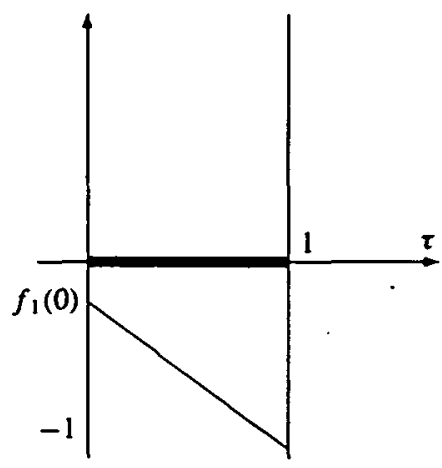

FIGURE 2. $f_{1}(0) \leq 0$.

3.2. Non-trivial equilibria. Now we discuss the existence and stability of the non-trivial equilibria. Note that if $Y_{2}^{*}=0$, then $H^{*}=0$, so that $G^{*}=c \beta_{1} Y_{1}^{*} / N^{*}$. Therefore $\left(G^{*}, 0\right)$ is a fixed point of $\Phi$ where

$$
G^{*}=\mu\left[\frac{c \beta_{1}(1-\tau)}{\mu}-1\right]=\mu\left(R_{1}-1\right),
$$

with $R_{1}$ defined in (3.4). Since $\mu$ is assumed positive, $G^{*}$ is positive whenever $R_{1}>1$. By substituting $G^{*}$ (above) and $H^{*}=0$ in the population densities, it is easy to see that the fixed point $\left(G^{*}, 0\right)$ of $\Phi$ corresponds to a non-trivial equilibrium (free of HIV and AIDS) given by

$$
E_{1}=\left[\frac{\Pi}{\mu R_{1}}, \frac{(1-\tau) \Pi\left(R_{1}-1\right)}{\mu R_{1}}, 0,0, \frac{\tau \Pi\left(R_{1}-1\right)}{\mu R_{1}}\right] .
$$

It should be noted that we require $\mathscr{R}>1(\mathscr{R}$ is the basic reproductive number of infection) to ensure the existence of a non-trivial equilibrium. It can be shown, after some manipulation, that the Jacobian of $\Phi$ at $\left(G^{*}, 0\right)$ is

$$
J_{1}=\left(\left.\begin{array}{cc}
D_{1} & \frac{\partial \phi_{1}(G, H)}{\partial H} \\
0 & D_{2}
\end{array}\right|_{\left(C^{*}, 0\right)}\right)
$$

where $D_{1}=1 / R_{1}, D_{2}=\left(R_{2} / R_{1}\right)\left[1+(1-\tau)\left(R_{1}-1\right)\right]$ and $R_{2}$ is as defined in (3.4).

The eigenvalues of $J_{1}$ are $D_{1}$ and $D_{2}$. Since $R_{1}>1$, it follows that $D_{1}<1$. Thus $E_{1}$ is locally asymptotically stable if $D_{2}<1$ and unstable if $D_{2}>1$.

Suppose now that $G^{*}=H^{*}$ (that is, $Y_{1}^{*}=0$ ). In this case, the fixed point of $\Phi$ is

$$
\left[\frac{(\mu+d)(\mu+v)\left(R_{2}-1\right)}{\mu+v+d}, \frac{(\mu+d)(\mu+v)\left(R_{2}-1\right)}{\mu+v+d}\right] .
$$


Thus $G^{*}$ and $H^{*}$ are positive if $R_{2}>1$. This fixed point corresponds to another non-trivial equilibrium (pathogen-free)

$$
\begin{aligned}
E_{2}= & {\left[\frac{(\mu+v+d) \Pi}{R_{2}(\mu+v)(\mu+d)-v d}, 0, \frac{(\mu+d) \Pi\left(R_{2}-1\right)}{R_{2}(\mu+v)(\mu+d)-v d},\right.} \\
& \left.\frac{v \Pi\left(R_{2}-1\right)}{R_{2}(\mu+v)(\mu+d)-v d}, 0\right] .
\end{aligned}
$$

It is worth mentioning that if $R_{2}>1$, then $R_{2}(\mu+v)(\mu+d)-v d>\mu(\mu+v+d)>0$. Thus none of the components of $E_{2}$ is negative for $R_{2}>1$.

The Jacobian of $\Phi$ at $\left(G^{*}, H^{*}\right)$ is given by

$$
J_{2}=\left(\begin{array}{cc}
\left.\frac{\partial\left(\phi_{2}+\phi_{3}\right)}{\partial G}\right|_{\left(G^{*}, H^{*}\right)} & \left.\frac{\partial\left(\phi_{2}+\phi_{3}\right)}{\partial H}\right|_{\left(G^{*}, H^{*}\right)} \\
\left.\frac{\partial \phi_{2}}{\partial G}\right|_{\left(G^{*}, H^{*}\right)} & \left.\frac{\partial \phi_{2}}{\partial H}\right|_{\left(G^{*}, H^{*}\right)}
\end{array}\right),
$$

where $\phi_{1}=\phi_{2}+\phi_{3}$ and $\phi_{1}$ and $\phi_{2}$ are defined as before. The characteristic polynomial of $J_{2}$ is

$$
\lambda^{2}-\left.\lambda\left(\frac{\partial \phi_{2}}{\partial H}+\frac{\partial \phi_{2}}{\partial G}+\frac{\partial \phi_{3}}{\partial G}\right)\right|_{\left(G^{*}, H^{*}\right)}+\left.\left(\frac{\partial \phi_{2}}{\partial H} \frac{\partial \phi_{3}}{\partial G}-\frac{\partial \phi_{2}}{\partial G} \frac{\partial \phi_{3}}{\partial H}\right)\right|_{\left(G^{*}, H^{*}\right)} .
$$

It is easy to see that

$$
\left.\frac{\partial \phi_{3}}{\partial H}\right|_{\left(G^{\bullet}, H^{\bullet}\right)}=-\left.\frac{\partial \phi_{3}}{\partial G}\right|_{\left(G^{*}, H^{\bullet}\right)} .
$$

Thus the roots of the characteristic polynomial of $J_{2}$ are

$$
\lambda_{1}=\left.\frac{\partial \phi_{3}}{\partial G}\right|_{\left(G^{*}, H^{*}\right)}=\frac{c \beta_{1}(1-\tau)}{\left((\mu+d)(\mu+v)\left(R_{2}-1\right) /(\mu+v+d)+\mu\right) R_{2}}
$$

and

$$
\lambda_{2}=\left.\left(\frac{\partial \phi_{2}}{\partial G}+\frac{\partial \phi_{2}}{\partial H}\right)\right|_{\left(G^{*}, H^{*}\right)}=\frac{1}{R_{2}} .
$$

Therefore $E_{2}$ is locally asymptotically stable if $\max \left\{\lambda_{1}, \lambda_{2}\right\}<1$.

It should be mentioned that another positive fixed point of $\Phi$, which corresponds to the coexistence of the two diseases (that is, $Y_{1} \neq 0, Y_{2} \neq 0$ ), exists. However, its closed-form expression could not be found, thereby making it impossible to analyse it qualitatively at this point. 




FIGURE 3. $f_{2}(0)=R_{2}-1>0$ and $\tau_{1}^{*}<\tau_{2}^{*}$.

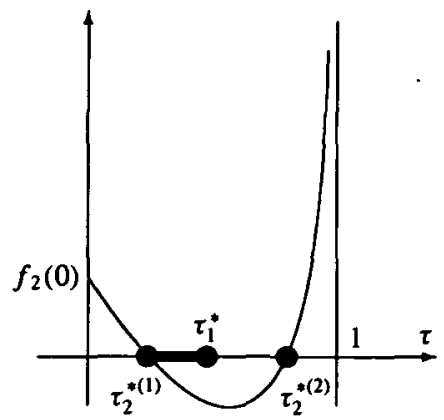

FIGURE 5. $f_{2}(\tau)<0$ for some $\tau \in(0,1)$ and $\tau_{2}^{*(1)}<\tau_{1}^{*}<\tau_{2}^{*(2)}$.

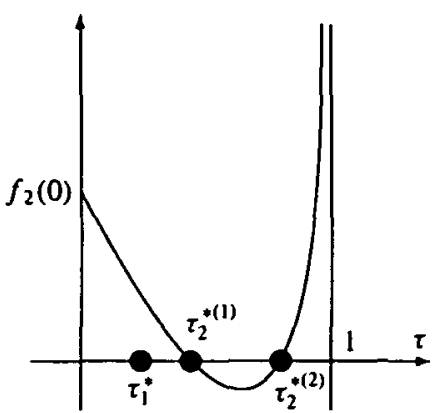

FIGURE 4. $f_{2}(\tau)<0$ for some $\tau \in(0,1)$ and $\tau_{1}^{*}<\tau_{2}^{*(1)}<\tau_{2}^{*(2)}$.

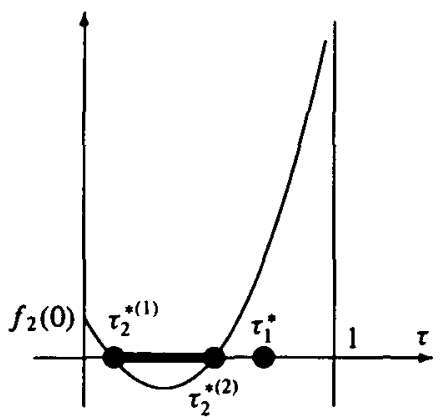

FIGURE 6. $f_{2}(\tau)<0$ for some $\tau \in(0,1)$ and $\tau_{2}^{*(1)}<\tau_{2}^{*(2)}<\tau_{1}^{*}$.

\section{Effect of the treatment rate ( $\tau$ )}

In this section, we shall discuss the effect of the treatment rate on the stability of the three equilibria.

4.1. Effect of $\tau$ on the stability of $E_{0}$. Without loss of generality, we assume that $R_{2}<1$ (otherwise $\mathscr{R} \geq 1$ and the disease-free equilibrium is unstable). Furthermore, let $f_{1}(\tau)=c \beta_{1}(1-\tau) / \mu-1$, for $0 \leq \tau \leq 1$. We consider the cases $f_{1}(0)>0$ and $f_{1}(0) \leq 0$.

Case 1. Suppose $f_{1}(0)=c \beta_{1} / \mu-1>0$. In this case, it is easy to see that there is a unique $\tau_{1}^{*}$ such that $f_{1}\left(\tau_{1}^{*}\right)=0$ and the region of the local asymptotic stability of $E_{0}$ is where $f_{1}(\tau)<0$. Thus $E_{0}$ is stable provided $\tau_{1}^{*}<\tau \leq 1$ (see Figure 1 ).

Case 2. Suppose $f_{1}(0) \leq 0$. Here $f_{1}(\tau)<0$ for every $\tau \in[0,1]$ (see Figure 2). Thus $R_{1}<1$. Hence $E_{0}$ is locally asymptotically stable. These results can be summarised in the following theorem. 
THEOREM 4.1. (a) If $c \beta_{1}>\mu$, then $E_{0}$ is locally asymptotically stable whenever $\tau_{1}^{*}<\tau<1$.

(b) If $c \beta_{1} \leq \mu$, then $E_{0}$ is locally asymptotically stable for every $\tau \in[0,1]$.

4.2. Effect of $\tau$ on the stability of $E_{1}$. Define now a function $f_{2}(\tau)$ given by

$$
f_{2}(\tau)=\frac{\mu R_{2}}{c \beta_{1}(1-\tau)}\left[1+(1-\tau)\left(\frac{c \beta_{1}(1-\tau)}{\mu}-1\right)\right]-1, \quad \text { for } \quad 0 \leq \tau<1 .
$$

We consider two cases as follows.

Case 1. Suppose $f_{2}(0)=R_{2}-1>0$. It is easy to see that $\lim _{\tau \rightarrow 1} f_{2}(\tau)=+\infty$. Further, there is a unique $\tau_{2}^{*}$ such that $f_{2}^{\prime}\left(\tau_{2}^{*}\right)=0$. If $f_{2}(\tau)>0$ for $\tau \in(0,1)$, then $D_{2} \geq 1$ for every $\tau \in[0,1]$ and hence $E_{1}$ is unstable (see Figure 3). Suppose that $f_{2}(\tau)<0$ for some $\tau \in(0,1)$. Then there exist $\tau_{2}^{*(1)}$ and $\tau_{2}^{*(2)}$ with $\tau_{2}^{*(1)}<\tau_{2}^{*(2)}$ such that $f_{2}\left(\tau_{2}^{*(1)}\right)=f_{2}\left(\tau_{2}^{*(2)}\right)=0$. If $\tau_{2}^{*(1)}>\tau_{1}^{*}$, then $D_{2}<1$ whenever $\tau \in\left(\tau_{2}^{*(1)}, \tau_{2}^{*(2)}\right)$. In this case, $D_{1}>1$ and thus $E_{1}$ is unstable (see Figure 4). If $\tau_{2}^{*(1)}<\tau_{1}^{*}$ and $\tau_{2}^{*(2)}>\tau_{1}^{*}$, then we need $\tau \in\left(\tau_{2}^{*(1)}, \tau_{1}^{*}\right)$ to establish the local asymptotic stability of $E_{1}$ (see Figure 5). In this case, if $\tau \in\left(\tau_{1}^{*}, \tau_{2}^{*(2)}\right)\left(D_{2}<1\right)$, then $D_{1}>1$ (because here $R_{1}<1$ ); thus $E_{1}$ is also unstable. Finally, if $\tau_{2}^{*(2)}<\tau_{1}^{*}$, then $E_{1}$ is locally asymptotically stable provided $\tau \in\left(\tau_{2}^{*(1)}, \tau_{2}^{*(2)}\right.$ ) (see Figure 6).

THEOREM 4.2. Suppose that $R_{2}>1$.

(a) If $f_{2}(\tau) \geq 0$ for $0<\tau<1$ or $\tau_{2}^{*(1)} \geq \tau_{1}^{*}$, then $E_{1}$ is unstable for every $\tau \in(0,1)$.

(b) If $\tau_{2}^{*(1)}<\tau_{1}^{*} \leq \tau_{2}^{*(2)}$, then $E_{1}$ is locally asymptotically stable whenever $\tau \in$ $\left(\tau_{2}^{*(1)}, \tau_{1}^{*}\right)$.

(c) If $\tau_{2}^{*(2)} \leq \tau_{1}^{*}$, then $E_{1}$ is locally asymptotically stable whenever $\tau \in\left(\tau_{2}^{*(1)}, \tau_{2}^{*(2)}\right)$.

Case 2. Suppose $f_{2}(0)=R_{2}-1 \leq 0$. In this case, there is a unique $\tau_{2}^{*(3)}$ such that $f_{2}\left(\tau_{2}^{*(3)}\right)=0$. If $\tau_{2}^{*(3)} \geq \tau_{1}^{*}$, then $D_{2}<1$ whenever $\tau \in\left(0, \tau_{2}^{*(3)}\right)$. Thus $E_{1}$ is locally asymptotically stable if $\tau \in\left(0, \tau_{1}^{*}\right)$ (see Figure 7). If $\tau_{2}^{*(3)}<\tau_{1}^{*}$, then $E_{1}$ is locally asymptotically stable whenever $\tau \in\left(0, \tau_{2}^{*(3)}\right)$ (see Figure 8 ).

THEOREM 4.3. Suppose that $R_{2}<1$.

(a) If $\tau_{2}^{*(3)} \geq \tau_{1}^{*}$, then $E_{1}$ is locally asymptotically stable whenever $\tau \in\left(0, \tau_{1}^{*}\right)$.

(b) If $\tau_{2}^{*(3)}<\tau_{1}^{*}$, then $E_{1}$ is locally asymptotically stable whenever $\tau \in\left(0, \tau_{2}^{*(3)}\right)$.

4.3. Effect of $\tau$ on the stability of $E_{2}$. Define

$$
f_{3}(\tau)=\frac{c \beta_{1}(1-\tau)}{\left((\mu+d)(\mu+v)\left(R_{2}-1\right) /(\mu+v+d)+\mu\right) R_{2}}-1, \quad \text { for } 0<\tau<1 .
$$




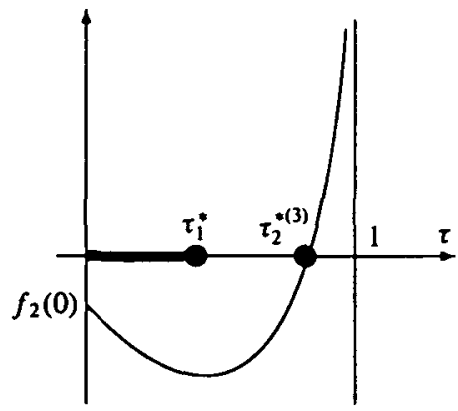

FIGURE 7. $f_{2}(0)=R_{2}-1 \leq 0$ and $\tau_{1}^{*} \leq \tau_{2}^{*(3)}$. FIGURE 8. $f_{2}(0)=R_{2}-1 \leq 0$ and $\tau_{2}^{*(3)} \leq \tau_{1}^{*}$.

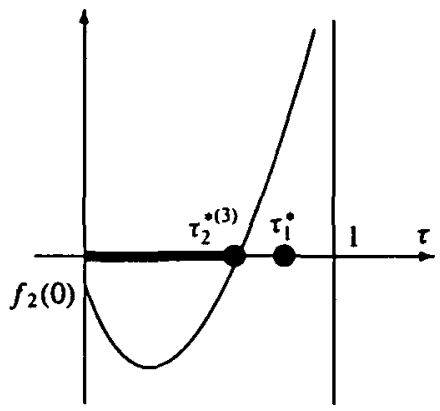

Notice that $f_{3}(\tau)<R_{1} / R_{2}-1$. It follows that if $R_{1}<1$, then $E_{2}$ is locally asymptotically stable, since $R_{2}>1$ ( (a necessary condition for the existence of $E_{2}$ ). Now we consider two cases as follows. If $f_{3}(0)>0$, then there exists a unique $\tau_{3}^{*}$ such that $f_{3}\left(\tau_{3}^{*}\right)=0$. Therefore $E_{2}$ is locally asymptotically stable if $\tau_{3}^{*}<\tau<1$ (that is, $f_{3}(\tau)<0$ or $\left.\lambda_{1}<1\right)$. Now suppose that $f_{3}(0) \leq 0$. Then $f_{3}(\tau)<0$ for every $\tau$ and thus $E_{2}$ is locally asymptotically stable for every $\tau \in[0,1]$.

THEOREM 4.4. (a) If $R_{1}<1$, then $E_{2}$ is locally asymptotically stable $\left(\right.$ for $\left.R_{2}>1\right)$. (b) If $f_{3}(0)>0$, then $E_{2}$ is locally asymptotically stable whenever $\tau \in\left(\tau_{3}^{*}, 1\right)$.

(c) If $f_{3}(0) \leq 0$, then $E_{2}$ is locally asymptotically stable for every $\tau$.

\section{Construction of a robust numerical method}

It should be noted that the five dependent variables of the model are populations and must therefore be non-negative. Thus any discrete model (numerical method) for approximating the model given by (2.1) must satisfy the "positivity" property of the model (see [6]). To construct a scheme with such a property, we use forwarddifference approximation for the derivatives in (2.1) and approximate the right-hand side functions appropriately as follows:

$$
\begin{aligned}
\frac{X^{n+1}-X^{n}}{\ell}= & \Pi-\mu X^{n+1}-\left(\frac{c \beta_{1} Y_{1}^{n}+c \beta_{2} Y_{2}^{n}}{X^{n}+Y_{1}^{n}+Y_{2}^{n}+A^{n}+Z^{n}}\right) X^{n+1}, \\
\frac{Y_{1}^{n+1}-Y_{1}^{n}}{\ell}= & \frac{c \beta_{1}(1-\tau)\left(2 Y_{1}^{n}-Y_{1}^{n+1}\right) X^{n+1}}{X^{n+1}+Y_{1}^{n}+Y_{2}^{n}+A^{n}+Z^{n}} \\
& -\frac{c \beta_{2} Y_{1}^{n+1} Y_{2}^{n}}{X^{n+1}+Y_{1}^{n}+Y_{2}^{n}+A^{n}+Z^{n}}-\mu Y_{1}^{n+1},
\end{aligned}
$$


190

$$
\begin{aligned}
& \frac{Y_{2}^{n+1}-Y_{2}^{n}}{\ell}= \frac{c \beta_{2} X^{n+1}\left(2 Y_{2}^{n}-Y_{2}^{n+1}\right)}{X^{n+1}+Y_{1}^{n+1}+Y_{2}^{n}+A^{n}+Z^{n}} \\
&+\frac{c \beta_{2} Y_{1}^{n+1}\left(2 Y_{2}^{n}-Y_{2}^{n+1}\right)}{X^{n+1}+Y_{1}^{n+1}+Y_{2}^{n}+A^{n}+Z^{n}}-(\mu+v) Y_{2}^{n+1}, \\
& \frac{A^{n+1}-A^{n}}{\ell}=v Y_{2}^{n+1}-(\mu+d) A^{n+1}, \\
& \frac{Z^{n+1}-Z^{n}}{\ell}= \frac{c \beta_{1} \tau Y_{1}^{n+1} X^{n+1}}{X^{n+1}+Y_{1}^{n+1}+Y_{2}^{n+1}+A^{n+1}+Z^{n}}-\mu Z^{n+1},
\end{aligned}
$$

where $X\left(t_{n}\right)=X^{n}, Y_{1}\left(t_{n}\right)=Y_{1}^{n}, Y_{2}\left(t_{n}\right)=Y_{2}^{n}, A\left(t_{n}\right)=A^{n}, Z\left(t_{n}\right)=Z^{n} ; n=$ $0,1,2, \ldots$ and $\ell>0$ is the time-step.

In (5.1b) and (5.1c), non-local implicit approximations for $Y_{1}$ and $Y_{2}$, given respectively by $Y_{1}^{n} \rightarrow 2 Y_{1}^{n}-Y_{1}^{n+1}$ and $Y_{2}^{n} \rightarrow 2 Y_{2}^{n}-Y_{2}^{n+1}$, have been used to ensure "positivity". Furthermore, although the equations in (5.1) are implicit in nature, they can be solved in sequence to give the following (Gauss-Seidel type) explicit formulation

$$
\begin{aligned}
X^{n+1} & =\frac{X^{n}+\ell \Pi}{1+\ell\left[\mu+\frac{c\left(\beta_{1} Y_{1}^{n}+\beta_{2} Y_{2}^{n}\right)}{X^{n}+Y_{1}^{n}+Y_{2}^{n}+A^{n}+Z^{n}}\right]}, \\
Y_{1}^{n+1} & =\frac{\left[1+\frac{2 \ell c \beta_{1}(1-\tau) X^{n+1}}{X^{n+1}+Y_{1}^{n}+Y_{2}^{n}+A^{n}+Z^{n}}\right] Y_{1}^{n}}{1+\ell\left[\frac{c \beta_{1}(1-\tau) X^{n+1}+c \beta_{2} Y_{2}^{n}}{X^{n+1}+Y_{1}^{n}+Y_{2}^{n}+A^{n}+Z^{n}}+\mu\right]}, \\
Y_{2}^{n+1} & \left.=\frac{Y_{2}^{n}+\frac{2 \ell c \beta_{2} Y_{2}^{n}\left(X^{n+1}+Y_{1}^{n+1}\right)}{X^{n+1}+Y_{1}^{n+1}+Y_{2}^{n}+A^{n}+Z^{n}}}{1+\frac{\ell c \beta_{2}\left(X^{n+1}+Y_{1}^{n+1}\right)}{X^{n+1}+Y_{1}^{n+1}+Y_{2}^{n}+A^{n}+Z^{n}}+\ell(\mu}+v\right) \\
A^{n+1} & =\frac{A^{n}+\ell v Y_{2}^{n+1}}{1+\ell(\mu+d)}, \\
Z^{n+1} & =\frac{Z^{n}+\frac{\ell \tau c \beta_{1} Y_{1}^{n+1} X^{n+1}}{X^{n+1}+Y_{1}^{n+1}+Y_{2}^{n+1}+A^{n+1}+Z^{n}}}{1+\ell \mu} .
\end{aligned}
$$

It can be seen from the right-hand sides of (5.2)-(5.6) that none of the methods admits negative terms for $0<\tau<1$. Thus the method $\{(5.2)-(5.6)\}$ satisfies the "positivity" requirement of the model (2.1). 
TABLE 1. Effect of $\tau$.

\begin{tabular}{||c|c|c|c|c|c|c|c|c|c||}
\hline$\tau$ & $R_{1}$ & $R_{2}$ & $D_{1}$ & $D_{2}$ & $\lambda_{1}$ & $\lambda_{2}$ & $Y_{1}^{*}$ & $Y_{2}^{*}$ & S.E. \\
\hline 0 & 6.451 & 0.392 & 0.155 & 0.392 & 91.306 & 2.550 & 54516 & 0 & $E_{1}$ \\
\hline 0.2 & 5.161 & 0.392 & 0.194 & 0.329 & 73.045 & 2.250 & 41613 & 0 & $E_{1}$ \\
\hline 0.4 & 3.871 & 0.392 & 0.258 & 0.276 & 54.783 & 2.250 & 28710 & 0 & $E_{1}$ \\
\hline 0.6 & 2.581 & 0.392 & 0.388 & 0.248 & 36.552 & 2.550 & 15807 & 0 & $E_{1}$ \\
\hline 0.8 & 1.290 & 0.392 & 0.775 & 0.322 & 18.261 & 2.250 & 2903 & 0 & $E_{1}$ \\
\hline 0.85 & 0.968 & 0.392 & 1.033 & 0.403 & 13.696 & 2.550 & 0 & 0 & $E_{0}$ \\
\hline
\end{tabular}

TABLE 2. Effect of $\beta_{2}$ (S.E. represents stable equilibrium).

\begin{tabular}{||c|c|c|c|c|c|c|c|c|c||}
\hline$\beta_{2}$ & $R_{1}$ & $R_{2}$ & $D_{1}$ & $D_{2}$ & $\lambda_{1}$ & $\lambda_{2}$ & $Y_{1}^{*}$ & $Y_{2}^{*}$ & S.E. \\
\hline 1 & 0.645 & 78.431 & 1.550 & 100 & $7.802 E-5$ & $1.275 E-5$ & 0 & 38844 & $E_{2}$ \\
\hline 0.8 & 0.645 & 62.745 & 1.550 & 80 & $1.220 E-4$ & $1.593 E-2$ & 0 & 38750 & $E_{2}$ \\
\hline 0.6 & 0.645 & 47.059 & 1.550 & 60 & $2.172 E-4$ & $2.125 E-2$ & 0 & 38594 & $E_{2}$ \\
\hline 0.4 & 0.645 & 31.373 & 1.550 & 40 & $4.900 E-4$ & $3.188 E-2$ & 0 & 38281 & $E_{2}$ \\
\hline 0.2 & 0.645 & 15.686 & 1.550 & 20 & $1.976 E-3$ & $6.375 E-2$ & 0 & 37331 & $E_{2}$ \\
\hline 0.1 & 0.645 & 7.843 & 1.550 & 10 & $8.041 E-3$ & 0.128 & 0 & 35382 & $E_{2}$ \\
\hline 0.01 & 0.645 & 0.784 & 1.550 & 1 & 1.160 & 1.275 & 0 & 0 & $E_{0}$ \\
\hline
\end{tabular}

\section{Numerical experiments}

In order to test the behaviour of the numerical scheme constructed above, numerous numerical simulations were carried out. The effect of the treatment parameter $\tau$ was monitored by simulating the method using the following parameter and initial values: $\Pi=2000, \mu=0.031, \beta_{1}=0.05, \beta_{2}=0.005, v_{2}=0.02, d=0.06, c=4$, $X^{0}=120,000, Y_{1}^{0}=10, Y_{2}^{0}=70,100, A^{0}=80,000, Z^{0}=30$. The results obtained, given in Table 1, are consistent with the theoretical predictions of Sections 3 and 4. As expected, higher values of $\tau$ lead to decreasing values of $Y_{1}^{*}$. In line with Theorem 4.1 (a), at $\tau=\tau_{1}^{*}=0.85$, we found that $Y_{1}^{*}=0$, implying that the pathogen can be eradicated provided at least $85 \%$ of the pathogen-infected population is effectively treated. This eradication condition coincides with the case where both $R_{1}$ and $R_{2}$ are less than unity (so that the disease-free equilibrium is stable).

Further numerical simulations were carried out to study the effect of $\beta_{2}$ on the dynamics of the HIV-infected population $Y_{2}$. The parameter and initial values used in these experiments were: $\Pi=2000, \mu=0.031, \beta_{1}=0.01, \tau=0.5, v=0.02$, $d=0.06, c=4, X^{0}=120,000, Y_{1}^{0}=10, Y_{2}^{0}=70,100, A^{0}=80,000, Z^{0}=30$. The results are depicted in Table 2, where it is evident that as $\beta_{2} \rightarrow 0, Y_{2}^{*} \rightarrow 0$. Thus, if an effective treatment mechanism is used to eliminate the competing pathogen from the population (making $Y_{1}=0$ ), then HIV eradication can be achieved in about 3 years (see Table 3 for the number of HIV infected individuals as a function of time) by (additionally) administering an active anti-retroviral therapy to the $\mathrm{HIV}$-infected 
TABLE 3. Dynamics of an HIV-infected population for $\beta_{2}=0.01$.

\begin{tabular}{||c|c|c|c|c|c|c||}
\hline Time (days) & 100 & 200 & 400 & 600 & 800 & 1000 \\
\hline HIV-infected population $\left(Y_{2}\right)$ & 5300 & 1375 & 140 & 16 & 2 & 0 \\
\hline
\end{tabular}

population that can reduce the probability of HIV transmission below 1\% (that is, make $\beta_{2} \leq 0.01$ ).

It should be mentioned that numerical simulations reveal that although $E_{1}$ and $E_{2}$ are both locally asymptotically stable (under the conditions of Theorems 4.2-4.4), neither of the two equilibria is globally asymptotically stable. Furthermore, in all the numerical experiments carried out, the numerical method gave profiles that converged only to the correct steady-state solutions and did not suffer any scheme-dependent instability for any set of parameter values used.

\section{Discussion and conclusion}

A new epidemic model has been developed and analysed for the interactions between HIV and a curable pathogen. Although pathogen-infected individuals are generally assumed to be more susceptible to HIV infection (in comparison to individuals who are not infected by the curable pathogen), our model assigns the same probability of HIV infection $\left(\beta_{2}\right)$ for both subpopulations $\left(X\right.$ and $\left.Y_{1}\right)$. The associated steady-state solutions of the model were found and analysed qualitatively. A novel finite-difference method, which is free of the scheme-dependent instabilities associated with the use of standard methods (such as the Euler and Runge-Kutta methods), was constructed and used to compute the solution of the model. Based on the parameter values used in our numerical simulations, the model predicts that the pathogen can be eradicated from the population if $85 \%$ of the pathogen-infected populace is effectively treated. Furthermore, with the pathogen eliminated, the model suggests that HIV eradication is feasible if an active anti-HIV therapy can reduce the HIV transmission parameter below a certain threshold. This threshold is $1 \%$ for the parameter values used in our simulations.

\section{Acknowledgements}

This work was supported in part by the Natural Sciences and Engineering Research Council of Canada (NSERC). One of the authors (S.M.M.) acknowledges the support of IIMS and the Department of Mathematics, University of Manitoba. The authors would like to thank the reviewers for their valuable comments which have improved the paper. 


\section{References}

[1] R. M. Anderson and R. M. May, Infectious diseases of humans (Oxford Univ. Press, London, 1991).

[2] S. Blower, K. Koelle, D. Kirschner and J. Mills, "Live attenuated HIV vaccines: predicting the trade-off between efficacy and safety", Proc. Natl. Acad. Sci. USA 98 (2001) 3618-3623.

[3] O. Diekmann, P. J. A. Heesterbeek and J. A. J. Metz, "On the definition and the computation of the basic reproduction ratio $R_{0}$ in models for infectious diseases in heterogeneous populations", $J$. Math. Biol. 28 (1990) 365-382.

[4] A. B. Gumel, T. D. Loewen, P. N. Shivakumar, B. M. Sahai, P. Yu and M. L. Garba, "Numerical modelling of the perturbation of HIV-1 during combination anti-retroviral therapy", Comput. Biol. Med. 31 (2001) 287-301.

[5] A. R. McLean and M. A. Nowak, "Models of interactions between HIV and other pathogens", J. Theoret. Biol. 155 (1992) 69-86.

[6] R. E. Mickens, Applications of non-standard finite-difference schemes (World Scientific, Singapore, 2000).

[7] A. S. Perelson and P. W. Nelson, "Mathematical analysis of HIV-1 dynamics in vivo", SIAM Rev. 41 (1999) 3-44.

[8] J. X. Velasco-Hernandez, "A model for Chagas disease involving transmission by vector and blood transfusion", Theoret. Population Biol. 46 (1994) 1-31. 